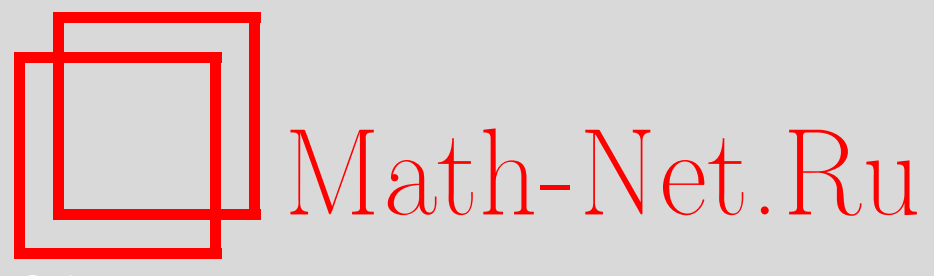

В. Е. Тараканов, Несколько замечаний об арифметических свойствах рекуррентных последовательностей на эллиптических кривых над конечным полем, Матем. заметки, 2007, том 82, выпуск 6, 926-933

DOI: https://doi.org/10.4213/mzm4192

Использование Общероссийского математического портала Math-Net.Ru подразумевает, что вы прочитали и согласны с пользовательским соглашением http://www.mathnet.ru/rus/agreement

Параметры загрузки:

IP : 3.81 .55 .215

26 апреля 2023 г., 12:54:52

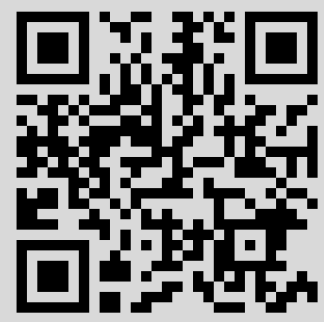


Том 82 выпуск 6 декабрь 2007

УДК 511

\section{Несколько замечаний об арифметических свойствах рекуррентных последовательностей на эллиптических кривых над конечным полем}

\section{В. Е. Тараканов}

В связи с проблемами в теории информации изучаются арифметические прогрессии, построенные на точках эллиптических кривых над конечным полем. Для одного из типов таких кривых устанавливается распределение квадратичных вычетов в соответствующих последовательностях $x$-координат точек прогрессии в случае, если кривая задана над простым полем. Приводится также некоторое описание множества всех прогрессий на эллиптических кривых над конечным полем.

Библиография: 11 названий.

Рекуррентные последовательности на эллиптических кривых над конечными полями в настоящее время интенсивно изучаются в связи с их приложениями в теории кодирования (см. [1]-[5]). Это обусловлено удобством вычисления элементов таких последовательностей и их хорошими статистическими свойствами. Здесь рассматривается один из видов таких последовательностей - арифметические прогрессии, которые могут использоваться при построении последовательностей псевдослучайных чисел. Мы установим для их элементов ряд свойств арифметического характера в случае, когда последовательность построена по эллиптическим кривым из некоторых классов, содержащих суперсингулярные эллиптические кривые (см. ниже).

Пусть $E=E_{a, b}$ - эллиптическая кривая (э.к.) над конечным полем $\mathscr{F}_{q}$ из $q$ элементов характеристики $p \neq 2,3$, заданная в нормальной форме Вейерштрасса:

$$
\begin{gathered}
E=E_{a, b}: \quad y^{2}=x^{3}+a x+b, \quad a, b \in \mathscr{F}_{q}, \\
4 a^{3}+27 b^{2} \neq 0 .
\end{gathered}
$$

Точками э.к. $E$ над произвольным полем $\mathscr{F}$ называются пары элементов $(x, y)$, принадлежащих какому-либо расширению поля $\mathscr{F}$ и удовлетворяющих (1); кроме того, точкой $E$ считается "бесконечно удаленная" точка $O$ (ей соответствует точка $(0,1,0)$ в проективном представлении $E$, отвечающем (1)). Если при этом $x, y \in \mathscr{F}$, то точка называется рациональной, или $\mathscr{F}$-точкой. Нас будут интересовать лишь рациональные точки, поэтому говоря о “точках на $E$ ”, мы будем подразумевать именно их. Точка $O$ считается рациональной для любой э.к.

Работа выполнена при поддержке программы "Ведущие научные школы" (грант № НШ4129.2006.1).

(C) B. Е. ТАраканов, 2007 
Как известно (см. [6]- [8]), точки $P=(x, y)$ э.к. $E$ из $(1), x, y \in \mathscr{F}_{q}$, вместе с $O$ образуют абелеву группу с нулем $O$ (в аддитивной записи) относительно некоторой операции композиции точек, которую мы обозначаем символом $\oplus$ (или просто + , если нет опасности ее смешения со сложением элементов в $\mathscr{F}_{q}$ ). Обратный элемент для $P=(x, y)-$ это

$$
\ominus P=(x,-y)
$$

Если $P_{1}=\left(x_{1}, y_{1}\right), P_{2}=\left(x_{2}, y_{2}\right)$ - точки на $E$, заданной уравнением $(1)$, и $P_{2} \neq \ominus P_{1}$, то $P_{1} \oplus P_{2}$ определяется как точка $P_{3}=\left(x_{3}, y_{3}\right)$ с

$$
x_{3}=k^{2}-x_{1}-x_{2}, \quad y_{3}=-\left(y_{1}+k\left(x_{3}-x_{1}\right)\right),
$$

где

$$
k=\left\{\begin{array}{lll}
\left(y_{2}-y_{1}\right)\left(x_{2}-x_{1}\right)^{-1} & \text { при } & x_{1} \neq x_{2}, \\
\left(3 x_{1}^{2}+a\right)\left(2 y_{1}\right)^{-1} & \text { при } & P_{1}=P_{2} ;
\end{array}\right.
$$

для любой точки $P \in E$ полагаем также

$$
P \oplus(\ominus P)=O, \quad P \oplus O=P .
$$

Группа точек эллиптической кривой имеет ранг не более 2, т.е. в каноническом представлении группа $E_{a, b}$ над $\mathscr{F}_{q}$ изоморфна прямой сумме циклических групп порядков $n_{1}$ и $n_{2}$ :

$$
E_{a, b} \cong Z_{n_{1}} \dot{+} Z_{n_{2}}, \quad n_{2} \mid n_{1}, \quad n_{1} n_{2}=n,
$$

где $n=\left|E_{a, b}\right|$ - порядок группы $E_{a, b}$; при этом также $n_{2} \mid(q-1)$ (см. [9]). Для приложений наибольший интерес представляет случай, когда $E_{a, b}$ - циклическая группа (т.е. когда $n_{2}=1$ ). Рассмотрим два класса э.к. частного вида:

$$
\begin{aligned}
E_{a}: & y^{2}=x^{3}+a x, & a \in \mathscr{F}_{q}, & a \neq 0, \\
E_{b}: & y^{2}=x^{3}+b, & b \in \mathscr{F}_{q}, & b \neq 0 .
\end{aligned}
$$

Для э.к. $E$ над $\mathscr{F}_{q}$ известно, что силовская $p$-подгруппа группы $E$ - это либо циклическая группа порядка $p$, либо единичная группа; в первом случае э.к. $E$ называется несуперсингулярной, а во втором - суперсингулярной. Суперсингулярные э.к. в большинстве случаев имеют циклическую группу точек (при $q \equiv 1(\bmod 4)$, $q=p^{t}, t$ нечетно, - всегда) (см. [10]). Обозначим $\left(\mathscr{F}_{q}^{*}\right)^{r}, r \geqslant 2$, подгруппу из всех $r$-х степеней элементов мультипликативной группы $\mathscr{F}_{q}^{*}$ поля $\mathscr{F}_{q}$. Рассмотрим на $\mathscr{F}_{q}^{*}$ квадратичный характер $\chi_{q}$ :

$$
\chi_{q}(c)= \begin{cases}1, & \text { если } c \in\left(\mathscr{F}_{q}^{*}\right)^{2}, \\ -1, & \text { если } c \notin\left(\mathscr{F}_{q}^{*}\right)^{2},\end{cases}
$$

доопределив его условием

$$
\chi_{q}(0)=0 .
$$

Нетрудно убедиться, что любая э.к. $E_{a}$ из $(6)$ при $q \equiv 3(\bmod 4)$ и $\chi_{q}(a)=1$, а также любая э.к. из $(7)$ при $q \equiv 2(\bmod 3)$ имеют порядок $q+1$. Следовательно, такие 
э.к. являются суперсингулярными: их группы точек циклические. Мы будем далее рассматривать рекуррентные последовательности, построенные на э.к., главным образом, с уравнениями (6) или (7).

Пусть $E$ - э.к. над $\mathscr{F}_{q}, P \neq O, I$ - две ее точки. Рекуррентная последовательность $S_{P, I}$ с отличными от $O$ элементами

$$
S_{P, I}(l+1)=S_{P, I}(l)+P, \quad l=1,2, \ldots,
$$

называется арифметической прогрессией на $E$ с начальным членом $I+P$ и разностью $P$. Последовательность (9) состоит не более, чем из $m$ членов, где $m-$ порядок точки $P$ (наименьшее целое $m>0$, для которого $m P=O$ ). Обозначим координаты точки $S_{P, I}(l)$ :

$$
x_{l}=x(I+l P), \quad y_{l}=y(I+l P) .
$$

С последовательностью (9) свяжем последовательность $x$-координат ее точек:

$$
x_{1}, \quad x_{2}, \ldots, \quad x_{l}, \ldots,
$$

где $x_{l}=x(I+l P), l=1,2, \ldots$. Прогрессии на точках эллиптических кривых над конечным полем и последовательности координат их точек впервые были рассмотрены А. М. Зубковым в связи с порождением псевдослучайных числовых последовательностей. Для целого $r$ назовем точку $Q \in E r$-делимой, если на $E$ существует точка $R$, для которой $r R=Q$. Например, для э.к. $E_{a, b}$ точка $O$ является 2-делимой, если многочлен в правой части (1) имеет корень $\xi \in \mathscr{F}_{q}$, так как точками порядка 2 являются, как известно, точки $(\xi, 0)$ (и только они).

Теорема 1. Пусть $E_{a}$ - эллиптическая кривая над полем $\mathscr{F}_{q}$ характеристики $p \neq 2,3$ с уравнением (6), $P \neq O, I$ - две точки на $E_{a}$. Пусть, далее, $S_{P, I}-$ прогрессия (9) на $E_{a}$ u

$$
x_{1}, \quad x_{2}, \ldots, \quad x_{l}, \quad \ldots
$$

- последовательность (10), образования $x$-координатами ее точек $I+l P, l=$ $1,2, \ldots$. Тогда справедливы следующие утверждения:

1) если $I$ - 2-делимая точка, то при любой $P \neq O$

$$
\chi_{q}\left(x_{l}\right)=1 \quad \text { или } \quad 0
$$

для всех четных $l$;

2) если $I+P-2$-делимая точка, то (11) выполнено для всех нечетных $l$;

3) если обе точки I и $P$ 2-делимы, то (11) выполнено при любом $l$.

ДокАзАтЕЛьство. Пусть сначала $E=E_{a, b}$ - произвольная эллиптическая кривая над полем $\mathscr{F}_{q}$ характеристики $p \neq 2,3$, заданная уравнением $(1)$ :

$$
y^{2}=g(x)=x^{3}+a x+b, \quad 4 a^{3}+27 b^{2} \neq 0 .
$$

Найдем необходимое и достаточное условие 2-делимости для ее точек. Пусть точка $Q=(u, v) \in E_{a, b} \quad 2$-делима, т.е. на $E_{a, b}$ существует точка $R=\left(u_{1}, v_{1}\right)$, для которой $2 R=Q$; при этом $v_{1} \neq 0$, так как в противном случае было бы $2 R=O \neq Q$. Согласно формуле (3) имеем

$$
u=k^{2}-2 u_{1}, \quad v=-\left(v_{1}+k\left(v-v_{1}\right)\right),
$$


где

$$
k=\left(3 u_{1}^{2}+a\right)\left(2 v_{1}\right)^{-1}
$$

Отсюда получается, что

$$
\left(3 u_{1}^{2}+a\right)^{2}=4 v_{1}^{2}\left(u+2 u_{1}\right)=4\left(u+2 u_{1}\right)\left(u_{1}^{3}+a u_{1}+b\right) .
$$

Из (12)-(14) следует, что координаты точки $R$ удовлетворяют уравнениям:

$$
\begin{gathered}
u_{1}^{4}-4 u u_{1}^{3}-2 a u_{1}^{2}-(4 a u+8 b) u_{1}+a^{2}-4 b u=0, \\
v=-\left(v_{1}+k\left(u-u_{1}\right)\right),
\end{gathered}
$$

где $k$ определяется формулой (13).

Обратно, пусть $Q=(u, v)$ - точка на $E_{a, b}$. Если $u_{1} \in \mathscr{F}_{q}$ - такое решение уравнения $(15)$, что $\chi_{q}\left(g\left(u_{1}\right)\right)=1$ и какой-либо из элементов $v_{1}= \pm \sqrt{g\left(u_{1}\right)}$ удовлетворяет равенству (16) с $k$, определенным в (13), то $Q=2 R$ для рациональной точки $R=\left(u_{1}, v_{1}\right)$ на $E_{a, b}$.

Запишем уравнение (15) в следующем виде:

$$
\left(u_{1}^{2}-a\right)^{2}-4 u\left(u_{1}^{3}+a u_{1}+b\right)=8 b u_{1} .
$$

Пусть теперь $E=E_{a}-$ э.к., заданная уравнением (6), т.е. $b=0$. Если $Q=(u, v)=2 R$, где $R=\left(u_{1}, v_{1}\right)$, то из (17) получаем, что

$$
4\left(u_{1}^{3}+a u_{1}\right) u=\left(u_{1}^{2}-a\right)^{2} .
$$

Так как $Q \neq O$, то $g\left(u_{1}\right)=u_{1}^{3}+a u_{1}=v_{1}^{2} \neq 0$, и мы приходим к равенству

$$
u=\left(u_{1}^{2}-a\right)^{2}\left(2 v_{1}\right)^{-2}
$$

Следовательно, в э.к. с уравнением (6) для любой 2 -делимой точки $Q \neq O$ имеем

$$
\chi_{q}(u)= \begin{cases}1, & \text { если } Q \neq(0,0), \\ 0, & \text { если } Q=(0,0)\end{cases}
$$

(последний случай возможен, очевидно, лишь тогда, когда $\chi_{q}(a)=1$ ).

Заметим теперь, что если $I-2$-делимая точка на $E_{a}$, то 2-делимой является любая точка $I+l P$ с четным $l$; если 2 -делима точка $I+P$, то 2 -делимой будет любая точка в прогрессии (9), имеющая нечетный номер $l$; наконец, если 2-делимы обе точки $I$ и $P$, то 2-делима любая точка в (9). Следовательно, по доказанному выше для соответствующих элементов $x_{l}=x(I+l P)$ в последовательности (10) во всех указанных случаях имеем $\chi_{q}\left(x_{l}\right)=1$ или 0 . Теорема доказана.

СледСтвиЕ. Если $E_{a}$ - эллиптическая кривая с уравнением (6) над простым полем характеристики $p \neq 2,3$ и $S_{P, I}$ - прогрессия (9) на $E_{a}$ с разностъю $P$ и начальным членом $I$, то при 2-делимой $I$ (2-делимой $I+P)$ в соответствующей последовательности (10) $x$-координат точек из $S_{P, I}$ каждый член $x_{l}$ с четным l (соответственно с нечетным l) есть квадратичный вычет по модулю $p$ (или нуль). 
В связи с теоремой 1 отметим, что условия 2-делимости для точек э.к., принадлежащих ее силовской 2-подгруппе, изучались в работах [9], [11].

Далее мы рассмотрим связи между элементами последовательностей (10) $x$-координат точек, принадлежащих прогрессиям (9), построенным для эллиптических кривых над конечным полем, заданных различными уравнениями (1). Напомним, что две э.к. $E$ и $E^{\prime}$ над полем $\mathscr{F}$ изоморфны, если существуют такие рациональные над $\mathscr{F}$ отображения $\varphi: E \rightarrow E^{\prime}$ и $\varphi^{-1}: E^{\prime} \rightarrow E$, определенные в каждой точке, что $\varphi \cdot \varphi^{-1}=\varphi^{-1} \cdot \varphi=\varepsilon$, где $\varepsilon-$ тождественное отображение; при этом группы точек э.к. $E$ и $E^{\prime}$ изоморфны: $E \cong E^{\prime}$.

Приведем несколько известных фактов об изоморфизмах эллиптических кривых над конечными полями (см. [7], [8]).

ПредложениЕ 1. а) Две э.к. над полем $\mathscr{F}_{q}$ характеристики $p \neq 2,3$ с уравнениями (1)

$$
E_{a, b}: \quad y^{2}=x^{3}+a x+b \quad u \quad E_{a^{\prime}, b^{\prime}}: \quad y^{2}=x^{3}+a^{\prime} x+b^{\prime}
$$

изоморфны над $\mathscr{F}_{q}$ тогда и только тогда, когда существует такой $u \in \mathscr{F}_{q}, u \neq 0$, что $a^{\prime}=u^{4} a, b^{\prime}=u^{6} b$; соответствующий изоморбизм задается следующим образом:

$$
P=(x, y) \rightarrow P^{\prime}=\left(u^{2} x, u^{3} y\right),
$$

где $P \in E_{a, b}, P^{\prime} \in E_{a^{\prime}, b^{\prime}}$.

б) Предположим, что $a \neq 0$. Тогда при $q \equiv 3(\bmod 4)$, а также при $q \equiv 1(\bmod 4)$, $b \neq 0$ существует $(q-1) / 2$ э.к., изоморфных $E_{a, b}$, а при $q \equiv 1(\bmod 4), b=0-$ $(q-1) / 4$ э.к., изоморфных $E_{a}=E_{a, 0}$. Если $a=0$, то существует не менее $(q-1) / 6$ э.к., изоморфньх $E_{b}=E_{0, b}$.

ПреДлОЖенИЕ 2. Имеется всего не более четырех классов э.к. над $\mathscr{F}_{q}$, состоящих из изоморфных над $\mathscr{F}_{q}$ э.к. с уравнением (6), и не более шести таких классов эллиптических кривых с уравнением (7).

Изоморфизм между двумя э.к. есть, очевидно, отношение эквивалентности. Оно порождает разбиение всего множества э.к. над $\mathscr{F}_{q}$ на $t$ подмножеств, каждое из которых состоит из изоморфных между собой кривых. Образуем множество

$$
\left\{E^{(1)}, E^{(2)}, \ldots, E^{(t)}\right\},
$$

состоящее из произвольно взятых э.к. в каждом из классов изоморфных э.к. над $\mathscr{F}_{q}$.

Теорема 2. Пусть Е - эллиптическая кривая над полем $\mathscr{F}_{q}$ характеристики $p \neq 2,3$ с уравнением (1) $и E^{\prime}$ - эллиптическая кривая, изоморфная $E$ над $\mathscr{F}_{q}$, $\varphi: E \rightarrow E^{\prime}$ - соответствуюший изоморфизм. Пусть, далее, на $E$ задана прогрессия $S_{P, I}$ (см. (9)) с разностью $P$ и начальным членом $I=O$, и пусть

$$
x(P), \quad x(2 P), \quad \ldots, \quad x(l P), \quad \ldots
$$

- соответствующая последовательность $x$-координат точек $S_{P, O}$. Тогда на $E^{\prime}$ существует такая точка $P^{\prime}$, что для последовательности х-координат точек про-

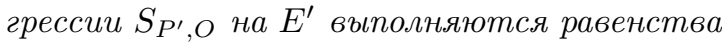

$$
x\left(l P^{\prime}\right)=c x(l P), \quad l=1,2, \ldots,
$$

где с-некоторый элемент $\mathscr{F}_{q}$, для которого $\chi_{q}(c)=1$. 
ДокАзАтельство. Рассмотрим каноническое разложение группы точек э.к. $E$ (см. (5)):

$$
E \cong Z_{n_{1}}+Z_{n_{2}}
$$

Пусть $G_{1}$ и $G_{2}$ - порождающие элементы группы $Z_{n_{1}}$ и соответственно $Z_{n_{2}}$. Точка $P$ представляется в виде

$$
P=t_{1} G_{1}+t_{2} G_{2}, \quad \text { где } \quad 0 \leqslant t_{1}<n_{1}, \quad 0 \leqslant t_{2}<n_{2} .
$$

Изоморфизм $\varphi$ индуцирует изоморфизм групп точек (обозначаемый той же буквой $\varphi$ ) на $E$ и на $E^{\prime}$. Поэтому для $G_{1}^{\prime}=\varphi\left(G_{1}\right)$ и $G_{2}^{\prime}=\varphi\left(G_{2}\right)$ имеем

$$
P^{\prime}=\varphi(P)=t_{1} G_{1}^{\prime}+t_{2} G_{2}^{\prime}, \quad 0 \leqslant t_{1}<n_{1}, \quad 0 \leqslant t_{2}<n_{2} ;
$$

при этом, очевидно, $G_{1}^{\prime}$ и $G_{2}^{\prime}$ - порождающие элементы циклических подгрупп порядков, соответственно $n_{1}$ и $n_{2}$ на $E^{\prime}$. Рассмотрим теперь прогрессию $S_{P^{\prime}, O}$ на $E^{\prime}$ и соответствующую ей последовательность (10) $x$-координат точек $S_{P^{\prime}, O}$ :

$$
x\left(P^{\prime}\right), \quad x\left(2 P^{\prime}\right), \quad \ldots, \quad x\left(l P^{\prime}\right), \quad \ldots
$$

Ясно, что

$$
\varphi(l P)=l \varphi(P)=l P^{\prime}, \quad l=1,2, \ldots
$$

Согласно предложению 1 (см. (18)) $x$-координаты точек изоморфных кривых при изоморфизме $\varphi$ связаны соотношением

$$
x(\varphi(Q))=u^{2} x(Q)
$$

для любой $Q \in E$ и некоторого $u \in \mathscr{F}_{q}, u \neq 0$. Таким образом, из (21) и (22), полагая $u^{2}=c$, получаем

$$
x\left(l P^{\prime}\right)=c x(l P), \quad l=1,2, \ldots
$$

с $\chi_{q}(c)=\chi_{q}\left(u^{2}\right)=1$. Теорема доказана.

СлеДСтвиЕ. Пусть $S_{P, O}$ - прогрессия (9) на ииклической э.к. $E=E_{a}$ с уравнением (6) или циклической э.к. $E=E_{b}$ с уравнением (7) над полем $\mathscr{F}_{q}$ характеристики $p \neq 2,3$ и $P \in E-$ точка максимального порядка $n$. Тогда можно задать несколько (не более четырех для $E=E_{a}$ и не более шести для $E=E_{b}$ ) последовательностей

$$
X^{\prime}=\left(x_{1}^{\prime}, x_{2}^{\prime}, \ldots, x_{n-1}^{\prime}\right),
$$

состоящих из $n-1$ элементов из $\mathscr{F}_{q}$, таких, что последовательность

$$
X=(x(P), x(2 P), \ldots, x(l P), \ldots)
$$

$x$-координат точек из $S_{P, O}$ двумя следующими преобразованиями получается из некоторой последовательности (23):

а) умножением каждого из членов (23) на один и тот же элемент $c\left(X^{\prime}, X\right) \in$ $\mathscr{F}_{q} c \chi_{q}\left(c\left(X^{\prime}, X\right)\right)=1$ 
б) перестановкой членов получающейся последовательности в соответствии с подстановкой их индексов:

$$
\left(\begin{array}{c}
l \\
l r
\end{array}\right), \quad l=1,2, \ldots, n-1,
$$

где $r$ - некоторое число, взаимно простое с $n$ (lr приводится по модулю $n)$.

ДокАЗАТЕЛЬСтво. Пусть $E=E_{a}$. Согласно предложению 2 имеется не более четырех классов изоморфных э.к. с уравнением (6). Возьмем в (19) э.к. $E^{\prime}$, изоморфную $E$, и обозначим через $\varphi$ соответствующее изоморфное отображение $E \rightarrow E^{\prime}$. На $E^{\prime}$ выберем произвольно порождающий элемент $Q$, зададим прогрессию $S_{Q, O}$ и, тем самым, последовательность

$$
X^{\prime}=(x(Q), x(2 Q), \ldots, x(l Q), \ldots)
$$

$x$-координат ее точек.

По теореме на $E^{\prime}$ существует такая точка $P^{\prime}$, что для некоторого $c \in \mathscr{F}_{q}$ с $\chi_{q}(c)=1$ имеем

$$
x\left(l P^{\prime}\right)=c x(l P), \quad l=1,2, \ldots,
$$

при этом $P^{\prime}=\varphi(P)$ (см. формулу $(20)$ ). Но $P^{\prime}$, будучи образом порождающего элемента, должна сама быть порождающим элементом группы $E^{\prime}$. Поэтому $P^{\prime}=r Q$ для некоторого $r$, взаимно простого с $n$, и мы имеем

$$
c x(l P)=x(\operatorname{lr} Q), \quad l=1,2, \ldots,
$$

где $\chi_{q}(c)=1$. Полагая $c\left(X^{\prime}, X\right)=c^{-1}$, находим, что последовательность $X$ действительно получается двумя указанными преобразованиями из последовательности (24). Ясно, что в качестве (24) может выступать одна из нескольких (не более, чем из 4-х) наперед заданных "стандартных" последовательностей (23), образованная элементами $x_{l}^{\prime}=x(l Q)-x$-координатами точек $l Q, l=1,2, \ldots$, прогрессии $S_{Q, O}$ на соответствующей э.к. $E^{\prime}$ из (19), изоморфной $E$, с разностью $Q$ - произвольным порождающим элементом группы $E^{\prime}$. В случае $E=E_{b}$ рассуждаем совершенно аналогично. Наше утверждение доказано.

Отметим, что аналогичное следствие может быть сформулировано и для э.к. общего вида (с $a, b \neq 0)$, однако в этом случае число классов изоморфных э.к. (и, соответственно, "базовых" последовательностей (23)) при больших $q$ весьма велико (близко к $2 q$, как следует из предложения 1).

\section{СПИСОК ЦИТИРОВАННОЙ ЛИТЕРАТУРЫ}

[1] G. Gong, T. Berson, D. Stinson, "Elliptic curve pseudorandom sequences generator", Selected Areas in Cryptography (Kingston, ON, 1999), Lecture Notes in Comput. Sci., 1758, Springer, Berlin, 2000, 34-48.

[2] G. Gong, C. Lam, "Linear recursive sequences over elliptic curves", Sequences and Their Applications (Bergen, 2001), Discrete Math. Theor. Comput. Sci. (Lond.), Springer, London, 2002, 182-196.

[3] C. P. Xing, "Constructions of sequences from algebraic curves over finite fields", Sequences and Their Applications (Bergen, 2001), Discrete Math. Theor. Comput. Sci. (Lond.), Springer, London, 2002, 88-100. 
[4] Н. Коблиц, Курс теории чисел и криптографии, ТВП, М., 2001.

[5] В.Е. Тараканов, “Линейные рекуррентные последовательности на эллиптических кривых и их применение в криптографии", Труды по дискретной математике, т. 9, Физматлит, М., 2006, 340-356.

[6] И.Р. Шафаревич, Основъ алгебраической геометрии, Наука, М., 1972.

[7] J.H. Silverman, J. Tate, Rational Points on Elliptic Curves, Undergraduate Texts in Math., Springer-Verlag, New York, 1992.

[8] J.H. Silverman, The Arithmetic of Elliptic Curves, Graduate Texts in Mathematics, 106, Springer-Verlag, New York, 1986.

[9] В.Е. Тараканов, "Свойства делимости точек эллиптических кривых над конечным полем", Труды по дискретной математике, т. 4, Физматлит, М., 2001, 243-258.

[10] R. Schoof, "Nonsingular plane curves over finite fields", J. Combin. Theory Ser. A, 46:2 (1987), 183-211.

[11] J. Miret, R. Moreno, A. Rao, M. Valis, "Determining the 2-Sylow subgroup of an elliptic curve over a finite field", Math. Comp., 74:249 (2005), 411-427.

В. Е. Тараканов

Поступило

Математический институт им. Стеклова РАН

20.01.2007 